\title{
Aplicaciones de la fibra óptica a la tecnología de los "Digital Twins" en estructuras.
}

\author{
* S. Villalba ${ }^{1}$, V. Alegre ${ }^{2}$, V. Ródenas ${ }^{3}$ \\ *Sergi Villalba Herrero: info@cotca.com \\ ${ }^{1}$ COTCA, S.A., UPC, Barcelona, España \\ ${ }^{2}$ COTCA, S.A., UPC, Barcelona, España \\ ${ }^{3}$ COTCA, S.A., Barcelona, España
}

\begin{abstract}
RESUMEN
La instrumentación de variables significativas en los puntos críticos de una estructura son ensayos de información sobre la respuesta real de la estructura.

Si se incorpora esa información al modelo inicial se está retroalimentando el modelo con el comportamiento real de la estructura. Ese proceso de incorporación de información tiene que ser de las variables importantes y en todos los puntos críticos que se considere. Con ello, se genera un prototipo que es un "gemelo digital" (Digital Twin) del comportamiento real de la infraestructura. De entre los muchos ensayos de información que permiten avanzar en la creación de "gemelos digitales" se presenta la fibra óptica que, midiendo microdeformaciones y temperatura en el tiempo y el espacio, es una buena herramienta aplicable al futuro "gemelo digital".
\end{abstract}

Palabras clave: fibra óptica, gemelo digital, microdeformaciones.

\begin{abstract}
The instrumentation of significant variables at the critical points of a structure are information tests on the real response of the structure.

If this information is incorporated into the initial model, the model is being fed back with the real behavior of the structure. This process of incorporating information must be one of the important variables and in all the critical points considered. With this, a prototype is generated that is a "digital twin" of the real behavior of my infrastructure.

Among the many information tests that allow progress in the creation of "digital twins", the optical fiber is presented, which by measuring microdeformations and temperature in time and space is a good tool applicable to the future "digital twin".
\end{abstract}

Key words: optical fiber, digital twin, microdeformations. 


\section{RESUMO}

A instrumentação de variáveis significativas nos pontos críticos de uma estrutura são testes de informação sobre a resposta real da estrutura.

Se essa informação for incorporada ao modelo inicial, o modelo está sendo realimentado com o comportamento real da estrutura. Este processo de incorporação de informações deve ser uma das variáveis importantes e em todos os pontos críticos considerados. Com isso, é gerado um protótipo que é um "gêmeo digital" do real comportamento da infraestrutura.

Entre os muitos testes de informação que permitem avançar na criação de "gêmeos digitais", apresenta-se a fibra óptica que, ao medir microdeformações e temperatura no tempo e no espaço, é uma boa ferramenta aplicável ao futuro "gêmeo digital".

Palavras-chave: fibra óptica, gêmeo digital, microdeformações.

\section{INTRODUCCIÓN A LA FIBRA ÓPTICA. LOS GEMELOS DIGITALES}

Cuando se está construyendo una estructura (un puente, una presa) cada vez es más necesario conocer el comportamiento real de la misma más allá del proyecto inicial. En una primera fase para tener el proyecto "as built" a partir de los ensayos de control, y más adelante para poder racionalizar los planes de mantenimiento y evaluar el riesgo con ensayos de información, que permiten obtener valores del comportamiento real de la estructura, o de las propiedades que nos interesan de la misma en momentos determinados a lo largo de su vida útil. El objetivo final siempre es el mismo, valorar el riesgo de daños a través del estudio de los estados límites últimos y de servicio de la infraestructura o de una parte de ella.

El riesgo se conocerá mejor si conocemos la respuesta real de la estructura a través de modelos, prototipos y algoritmos que representen la realidad en tiempo real. Estos modelos se mejoran instrumentando en el tiempo y en el espacio las zonas críticas y clave. Con ello se es capaz de conocer el comportamiento de la estructura a través de su historia de cargas, porque se mide la respuesta de la estructura o infraestructura a las acciones y a las condiciones de contorno que se tienen instrumentadas de forma continua o semicontinua en el tiempo.

En las estructuras modernas se puede avanzar en la creación de un modelo digital con toda la "historia de cargas" desde el principio de la obra. A medida que se gestiona la obra y se van midiendo "in situ" sus variables fundamentales, se puede mejorar el modelo inicial retroalimentándolo al incorporar los datos de la respuesta real y creando un "gemelo digital" del comportamiento de la estructura que, a su vez, permitirá simular situaciones de riesgo ante acciones singulares y conocer su respuesta. De esta forma podemos adelantarnos a la situación, por ejemplo, de acciones extraordinarias, porque al evaluar el riesgo puedo establecer un plan de contingencias que avise de un riesgo excesivo.

Una herramienta muy apta para estudiar las microdeformaciones, tensiones y variaciones térmicas en la estructura son los sistemas de fibra óptica (SFO). La fibra óptica está basada en el principio de propagación de la luz a través de un medio (cable de aleación de cristales de sílice).

Mediante la manipulación de factores ambientales que influyen en las propiedades de la luz que viaja a través de la fibra, ésta puede ser utilizada como medio para detectar e incluso medir perturbaciones externas en forma discreta o distribuida.

Las tecnologías de detección más conocidas y sus principales características se adjuntan en la Tabla 1 .

Tabla 1. Tecnologías de detección. 


\begin{tabular}{ccccc}
\hline $\begin{array}{c}\text { Tecnología } \\
\text { de sensor }\end{array}$ & $\begin{array}{c}\text { Tipo de } \\
\text { transductor }\end{array}$ & $\begin{array}{c}\text { Rango de } \\
\text { sensor }\end{array}$ & $\begin{array}{c}\text { Resolución } \\
\text { espacial }\end{array}$ & $\begin{array}{c}\text { Principales } \\
\text { medidas }\end{array}$ \\
\hline $\begin{array}{c}\text { Raman } \\
\text { OTDR }\end{array}$ & Distribuido & $\begin{array}{c}1 \mathrm{~km} \mathrm{[73]} \\
37 \mathrm{~km} \mathrm{[74]}\end{array}$ & $\begin{array}{c}1 \mathrm{~cm} \mathrm{[73]} \\
17 \mathrm{~m} \mathrm{[74]}\end{array}$ & Temperatura \\
\hline BOTDR & Distribuido & $20-50 \mathrm{~km}$ & $\approx 1 \mathrm{~m}$ & $\begin{array}{c}\text { Temperatura y } \\
\text { deformación }\end{array}$ \\
\hline BOTDA & Distribuido & $150-200 \mathrm{~km} \mathrm{[13]}$ & $\begin{array}{c}2 \mathrm{~cm}(2 \mathrm{~km})[75] \\
2 \mathrm{~m}(150 \mathrm{~km})[76]\end{array}$ & $\begin{array}{c}\text { Temperatura y } \\
\text { deformación }\end{array}$ \\
\hline $\begin{array}{c}\text { Rayleigh } \\
\text { OFDR/OBR }\end{array}$ & Distribuido & $50-70 \mathrm{~m} \mathrm{[11]}$ & $\approx 1 \mathrm{~mm}[66]$ & $\begin{array}{c}\text { Temperatura y } \\
\text { deformación }\end{array}$ \\
\hline FBG & Semi-distribuido & $\approx 100$ canales & $\begin{array}{c}2 \mathrm{~mm}(\text { longitud de } \\
\text { Bragg })[21]\end{array}$ & $\begin{array}{c}\text { Temperatura, } \\
\text { deformación y } \\
\text { desplazamiento }\end{array}$ \\
\hline
\end{tabular}

En la anterior tabla se observa la presencia de sensores basados en el dominio del tiempo (Raman Brillouin) y otros (Rayleigh) en el de la frecuencia. El tipo de transductor puede ser distribuido o semi-distribuido. Los rangos de detección van de 50m (Rayleigh) con varios canales desde un multiplexador a $150 \mathrm{~km}$ (Brillouin). La resolución espacial es de $1 \mathrm{~mm}$ para Rayleigh y de metros o centímetros para Brillouin. Y la precisión en sensores distribuidos de Rayleigh son $2 \mu \varepsilon$ y $0,2^{\circ} \mathrm{C} \mathrm{y}$ en Brillouin en el mejor de los casos actualmente $17 \mu \varepsilon$ y $2^{\circ} \mathrm{C}$.

Cada sistema tiene su campo de aplicación y, por ejemplo, para detectar fisuras el más indicado es el tipo OFDR/OBR de Rayleigh, cuya tecnología ha sido la empleada en el caso de este artículo. En ensayos de laboratorio (1) se ha podido comprobar que una vez adherida la fibra a la estructura puede ayudar o prevenir daños al detectar la aparición de fisuras llegando a indicar aberturas de $2 \mathrm{~mm}$ antes de desacoplarse.

Una vez instrumentada con fibra óptica una viga de un puente o una sección de un túnel o una zona considerada crítica en una estructura, por ejemplo, una fisura, se pueden hacer lecturas continuas o lecturas en el tiempo cuando se considera necesario porque la acción que se va a realizar sobre la estructura lo requiere, o porque queremos conocer la respuesta real ante acciones extraordinarias. La fibra puede también quedar embebida en el hormigón, pudiendo leer microdeformaciones cuando se considere tanto en el tiempo como en el espacio donde la hemos colocado.

Si se detectan los problemas con antelación se podrán resolver más rápidamente. Al detectar la fisura, se está avisando de una posible disfunción que tu "gemelo digital" puede tener contemplado como aviso, alerta o alarma en el plan de contingencias de la estructura.

El gemelo digital, en los puntos de la estructura donde está instrumentado es una réplica virtual realizada a imagen y semejanza de la estructura, midiendo los parámetros importantes en las secciones críticas tanto en el proceso de ejecución como durante la puesta en servicio. Con un "gemelo digital" que incorpore datos en tiempo real captados a través de sensores como la fibra óptica, acelerómetros, etc., se puede prever el comportamiento del puente, presa, túnel, a partir de, por ejemplo, su respuesta en secciones críticas.

Podemos predecir virtualmente todo lo que sucederá en el mundo físico y mejorar a partir del comportamiento conocido en tiempo real de las secciones críticas la respuesta de la estructura cuando sea necesario (2). 


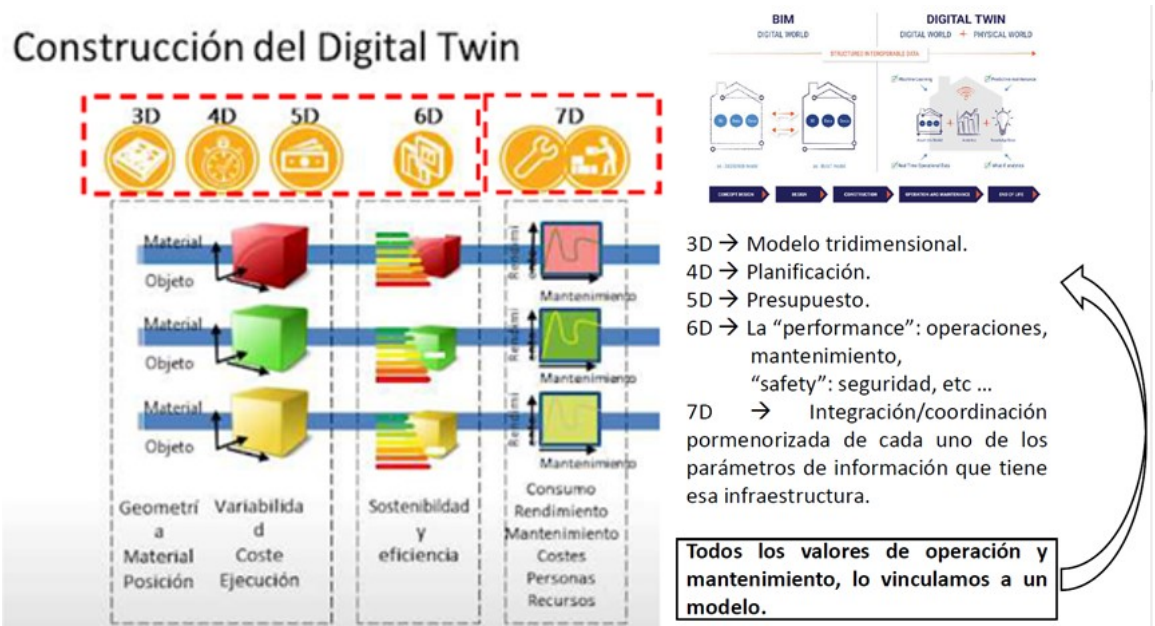

Figura 1. Introducción a la tecnología DT (Digital Twin).

En las aplicaciones aquí explicadas no se llega más que a las 4 de las 7 dimensiones que supone un "digital twin" completo. Es decir, los ejemplos de aplicación no incorporan la planificación (4), presupuesto (5), ni su consideración conjunta (6), pero sí se retroalimenta con los ensayos de información e instrumentación (solo con la información de la estructura) para tener al día el DT (7), y valorar el riesgo de la estructura a partir de su comportamiento real.

\section{APLICACIÓN A UNA TORRE DE REFRIGERACIÓN}

La torre de refrigeración estudiada es un hiperboloide parabólico de $120 \mathrm{~m}$ de altura con una lámina de $14 \mathrm{~cm}$, un anillo superior y otro inferior apoyado sobre pilares y una cimentación. La vida útil empieza con la puesta en funcionamiento en 1972. Se dispone de la información que en 1987-88 se hace una actuación de durabilidad. En 2007 y 2009 se acelera la evolución de los daños con la aparición de una grieta principal sensiblemente vertical de $70 \mathrm{~m}$ y otra secundaria. Se hace un estudio de daños y una digitalización de la torre y se modeliza el comportamiento, se define el estado actual y tras el estudio de daños con los ensayos de información correspondientes que indican el peso de las variables importantes (viento, durabilidad, terreno, geometría, ...) se proponen unas operaciones que dan lugar a un proyecto de reparación de carácter urgente y otras de seguimiento.

\section{Central Térmica de Puertollano (2007 - 2012)}

Características geométricas y dimensiones de la torre:

$\checkmark$ Altura total: $120 \mathrm{~m}$.

$\checkmark$ Altura pilares: $7.65 \mathrm{~m}$.

$\checkmark$ Número pilares: 88 diagonales.

$\checkmark$ Diámetro base de Torre: $87.36 \mathrm{~m}$.

$\checkmark$ Diámetro coronación: $54.49 \mathrm{~m}$.

$\checkmark$ Diámetro garganta: $48.00 \mathrm{~m}$.

$\checkmark$ Espesor medio lámina de revolución: $0.14 \mathrm{~m}$.

$\checkmark$ Viga coronación: $1.00 \times 0.30 \mathrm{~m}$.

$\checkmark$ Anillo inferior (viga de unión entre pilares): 0.75x.45 m. $\checkmark$ Hormigón: fck : $225 \mathrm{~kg} / \mathrm{cm} 2$.

$\checkmark$ Acero de armar: B 400S.

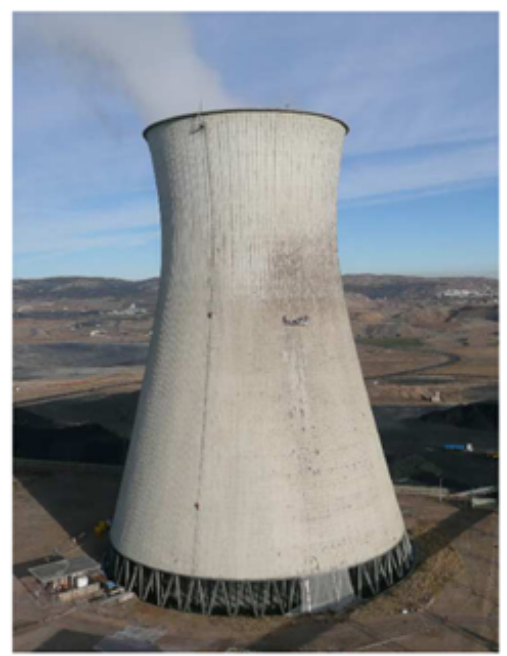

Figura 2. Aplicación a una torre de refrigeración. 
Para el seguimiento, entre los muchos ensayos de información que se realizan (resistencia del hormigón, porosidad, comportamiento dinámico, topografía, escáner, ...), se coloca la fibra óptica en la garganta y en la fisura principal (3). Se hizo un seguimiento recogiendo información de la fibra (Figura 3), la topografía, los acelerómetros, etc., durante 8 meses (Figura 4 y Figura 4. Lecturas de topografía.

). En lo que se refiere a la fibra, las microdeformaciones medidas, como se conoce la ecuación constitutiva del hormigón, dan tensiones en las secciones críticas estudiadas, que son la grieta principal y la garganta. Ahora se puede retroalimentar el modelo de comportamiento ya que no solo se tiene información de tensiones, sino que se han hecho los END (ensayos no destructivos) (¿Error! No se encuentra el origen de la referencia.) como un escáner con la geometría real que en la zona dañada no es un hiperboloide. También se mide topográficamente la evolución de los movimientos y mediante una acción indirecta se incorpora en el modelo. Ahora se está en condiciones de predecir mejor el comportamiento porque se conoce mejor el estado actual.

\section{Retroalimentación al MODELO

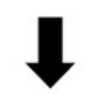 \\ Retroalimentación continua durante 8 MESES}

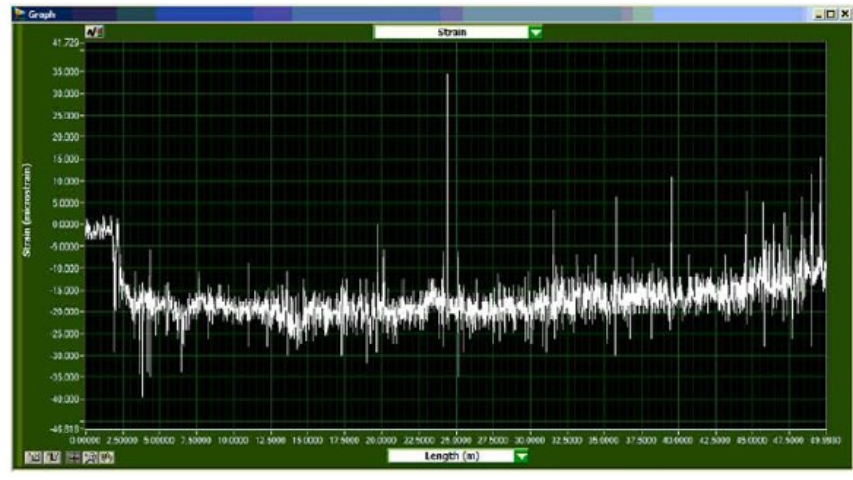

Figura 3. Seguimiento de la fibra óptica.

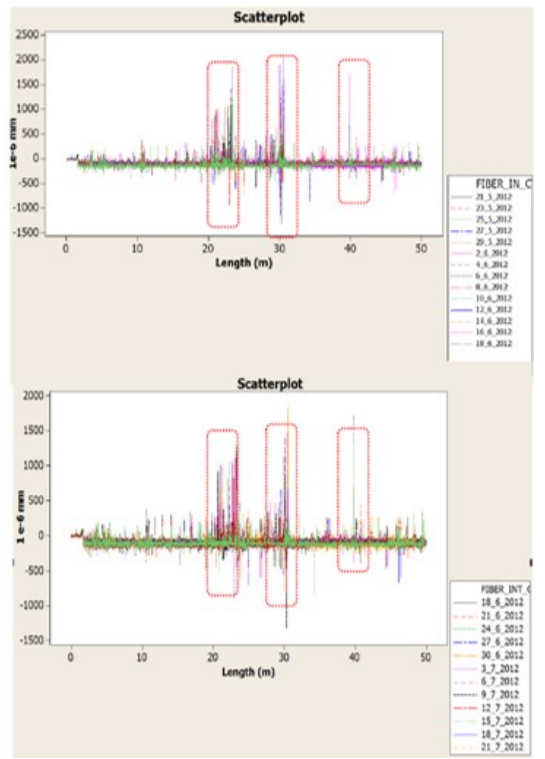

Optica.
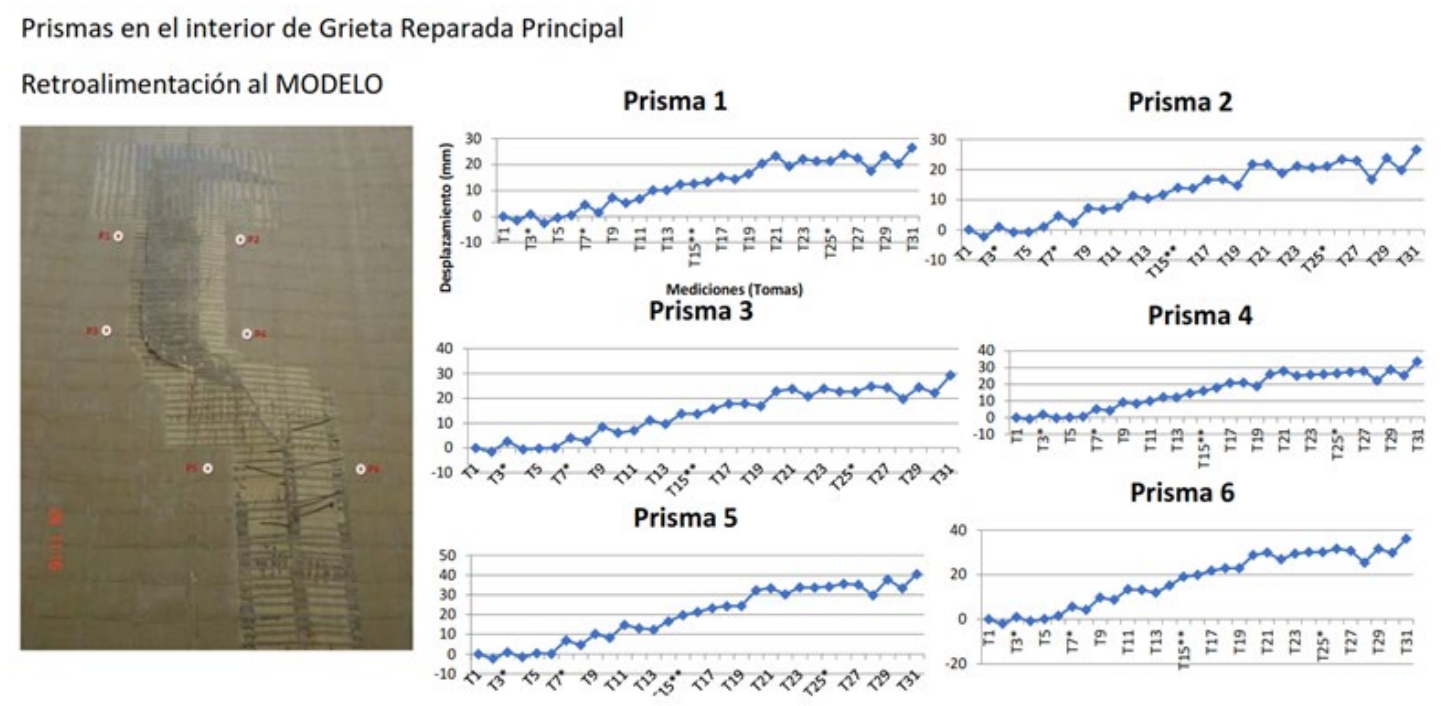

Figura 4. Lecturas de topografía. 


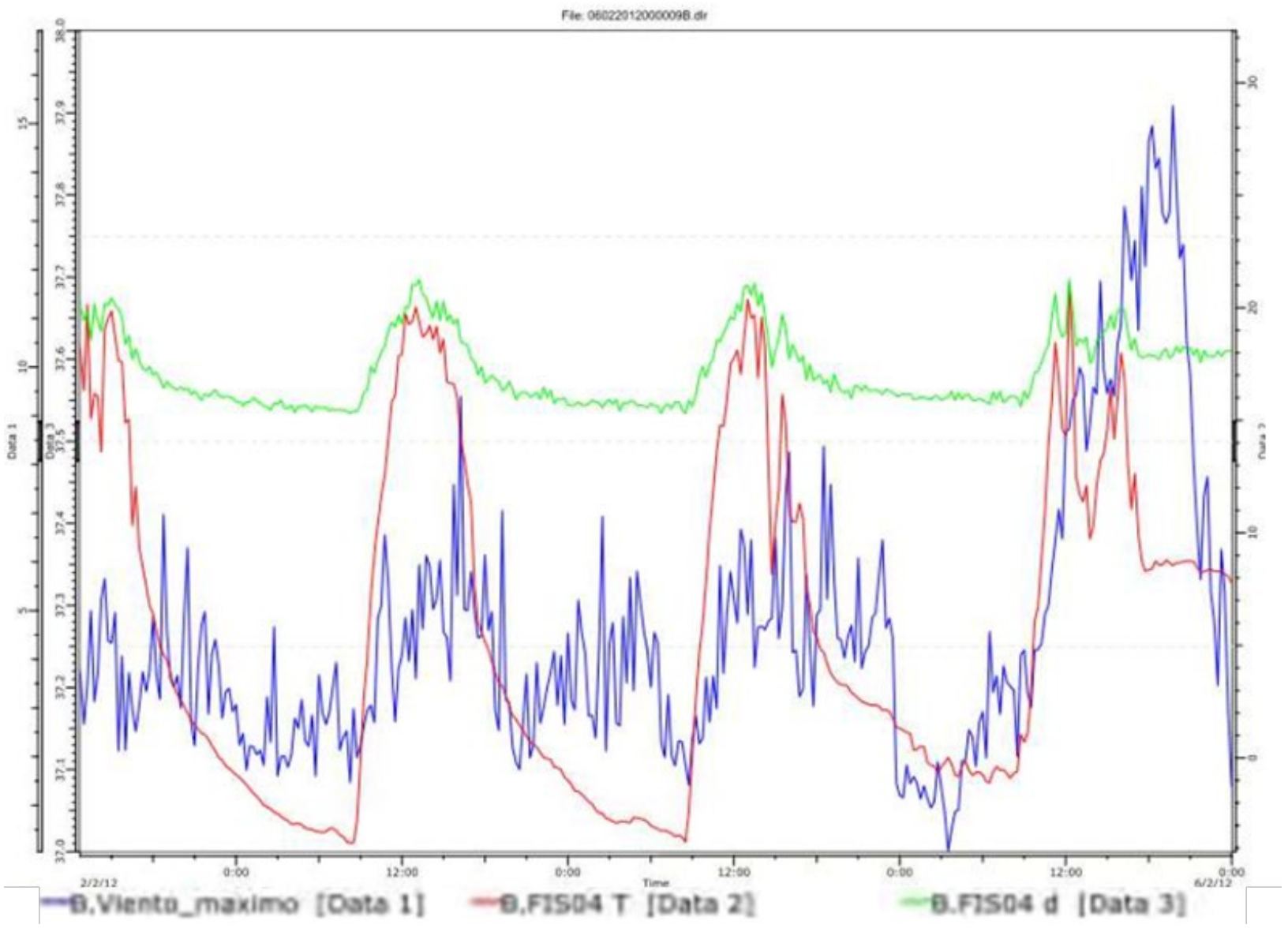

Figura 5. Lecturas de acelerómetros, fisurómetros, termómetros embebidos.

Implementación del MODELO 3D.F.E.M.

ENSAYOS REALIZADOS

1. Ensayos de resistencia del hormigón (7 testigos).

2. Ensayos en el acero corrugado (3 barras).

3. Espesores de carbonatación.

4. Ensayos de densidad, porosidad y absorción de agua (12 muestras).

5. Muestreo de espesores de pared (11).

6. Ensayos de difracción del hormigón (3).

7. Ensayos de agresividad del agua.

8. Ensayo de penetración de agua (1).

9. Ensayo del revestimiento activo.

10. Ensayos de roseta.

11. Ensayos de dianas entorno a la grieta.

12. Estudio del terreno.

13. Acelerómetros para descartar el efecto de unas voladuras cercanas.

14. Escáner de la geometría deformada.

La existencia de daños estructurales locales y de durabilidad generales, afectan a las condiciones de seguridad de la torre de refrigeración

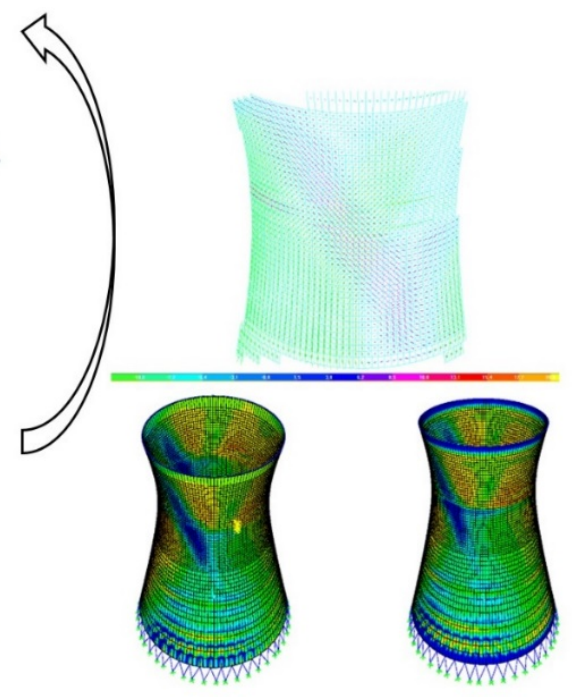

Figura 6. Ensayos END realizados. 
Si se tiene el comportamiento en tiempo real y se traspasa al modelo, en este caso manualmente, se puede calibrar el DT y valorar el riesgo con mayor certidumbre.

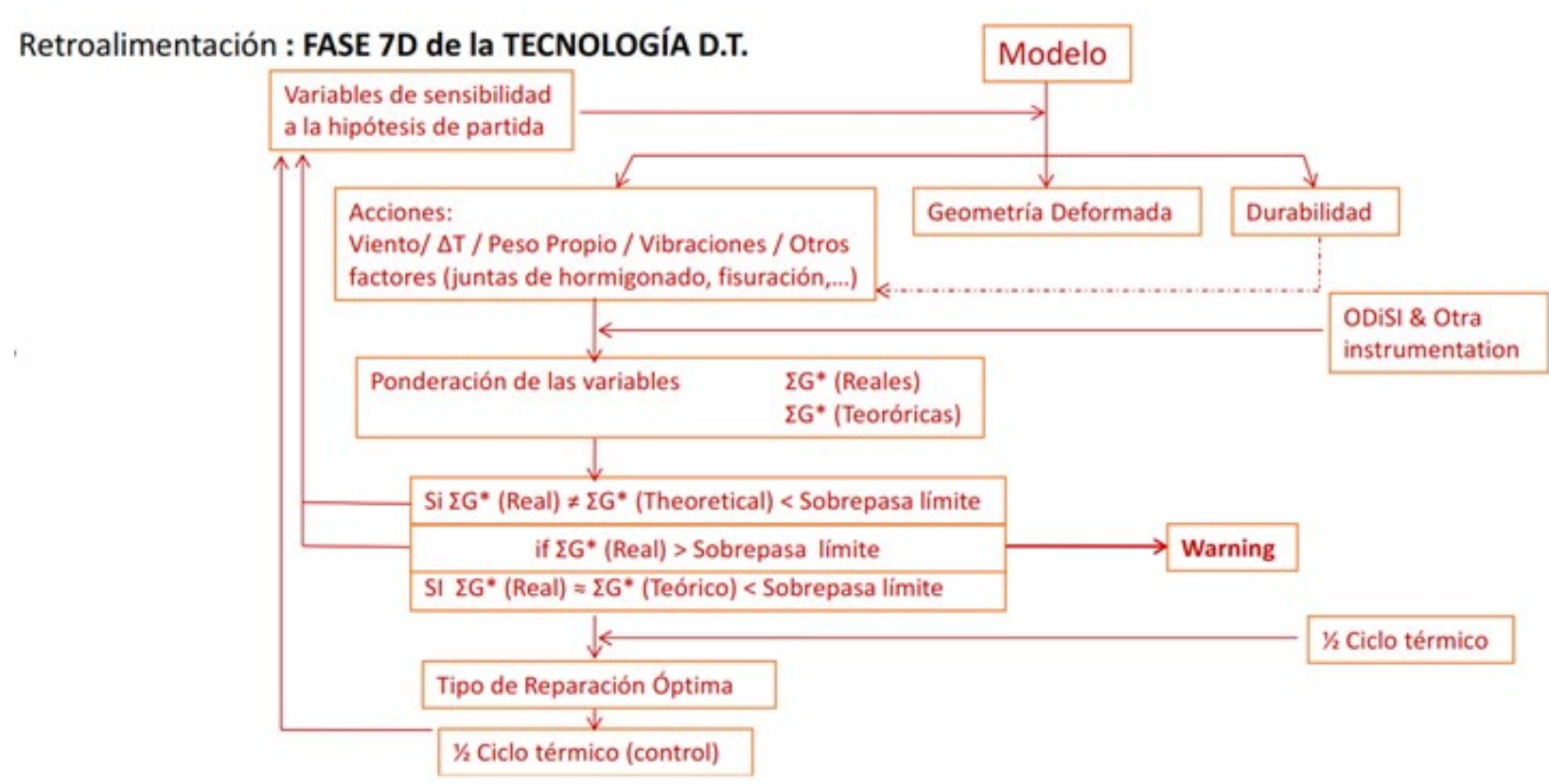

Figura 7. Retroalimentación del modelo.

Con esta nueva ponderación de pesos reales, rigideces de la reparación y estudios de sensibilidad, se puede optimizar la reparación de acuerdo con el modelo hipercalibrado. Véase el proceso de retroalimentación en este caso (Figura 7).

El nuevo proyecto de reparación de 2012 mejoraba la solución planteada de forma urgente en 2008 porque se implementaba toda la información de ensayos e instrumentación, en el DT creado de la torre.

\section{APLICACIÓN A UN TÚNEL DE LA LÍNEA 9 DEL METRO DE BARCELONA}

En Transports Metropolitans de Barcelona (TMB), se hizo en 2002 una definición del estado de la red de metro que, entre otros trabajos, dejó un túnel virtual de los entonces $92 \mathrm{~km}$ de red. Esto se realizó con un láser escáner que, montado en un vehículo y direccionado perpendicularmente a la dirección de la marcha, proporcionaba la sección transversal y la imagen real digitalizada (4). Si ahora, 20 años después, instrumentamos la sección transversal con fibra óptica podemos, por ejemplo, ver qué ovalización se ha producido en estos últimos 20 años en esa sección y actuar en consecuencia.

En el ejemplo presentado, de incorporación de ensayos de información para crear un modelo digital, no existe la información de los 20 años al ser una línea nueva, pero si se instrumenta ahora se tendrá información a incorporar en un futuro "gemelo digital" que incorpore todo lo instrumentado en secciones críticas de ahora en adelante.

La Línea 9 Norte de Barcelona consta de 8 estaciones con una longitud de línea de 7,8km con una durada media de trayecto de 15 minutos. Se presenta un ejemplo de un proceso completo de instrumentación con fibra óptica durante 29 meses de un túnel sobre el que se coloca un edificio. La instrumentación de la sección crítica ha permitido validar el modelo que se realizó y, lo que es 
más relevante, calibrar lo con la respuesta real de cada una de las operaciones generales llevadas a cabo sobre el túnel, y todas las intermedias que se han ido midiendo. Ello permite avanzar en la creación del gemelo digital, que no es de todo el túnel, pero sí de ese tramo instrumentado. El tramo instrumentado corresponde a la Vía 2 entre las paradas de Bon Pastor y Onze de Setembre. La monitorización de la Fibra Óptica (FO) se ha ubicado en el punto estratégico de influencia y afectación por la construcción del edificio I1 en el Barrio del Bon Pastor de Barcelona.

El túnel instrumentado de la L9 es una sección típica con vías superpuestas y losa intermedia. La fibra óptica se ha ubicado en el perímetro de la sección transversal, siempre de acuerdo con los criterios y/o indicaciones de la Propiedad. La Figura 8 muestra su colocación. La colocación de la fibra se ha llevado a cabo siguiendo el perímetro de una sección transversal en la posición indicada en planta.
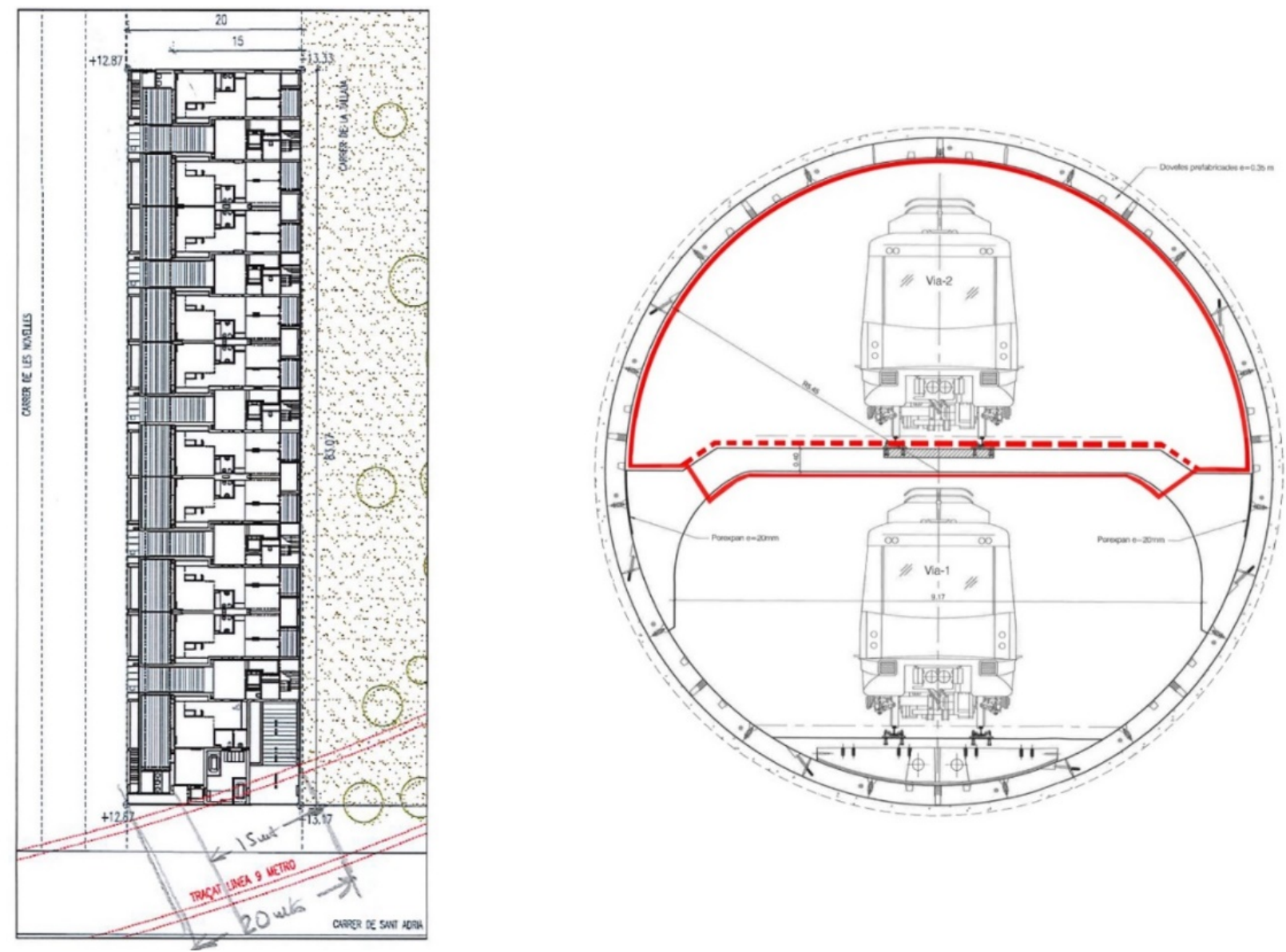

Figura 8. Planta de detalle del edificio en relación con el túnel del metro y sección del trazado de la fibra óptica.

La instrumentación planteada mide microdeformaciones y temperatura en la sección transversal del túnel, es decir, los posibles movimientos o disfunciones de la sección transversal.

En la lectura inicial de ceros con la fibra óptica se hace un catálogo de las disfunciones actuales (fisuras, humedades, coqueras, rugosidades anómalas, etc.). 


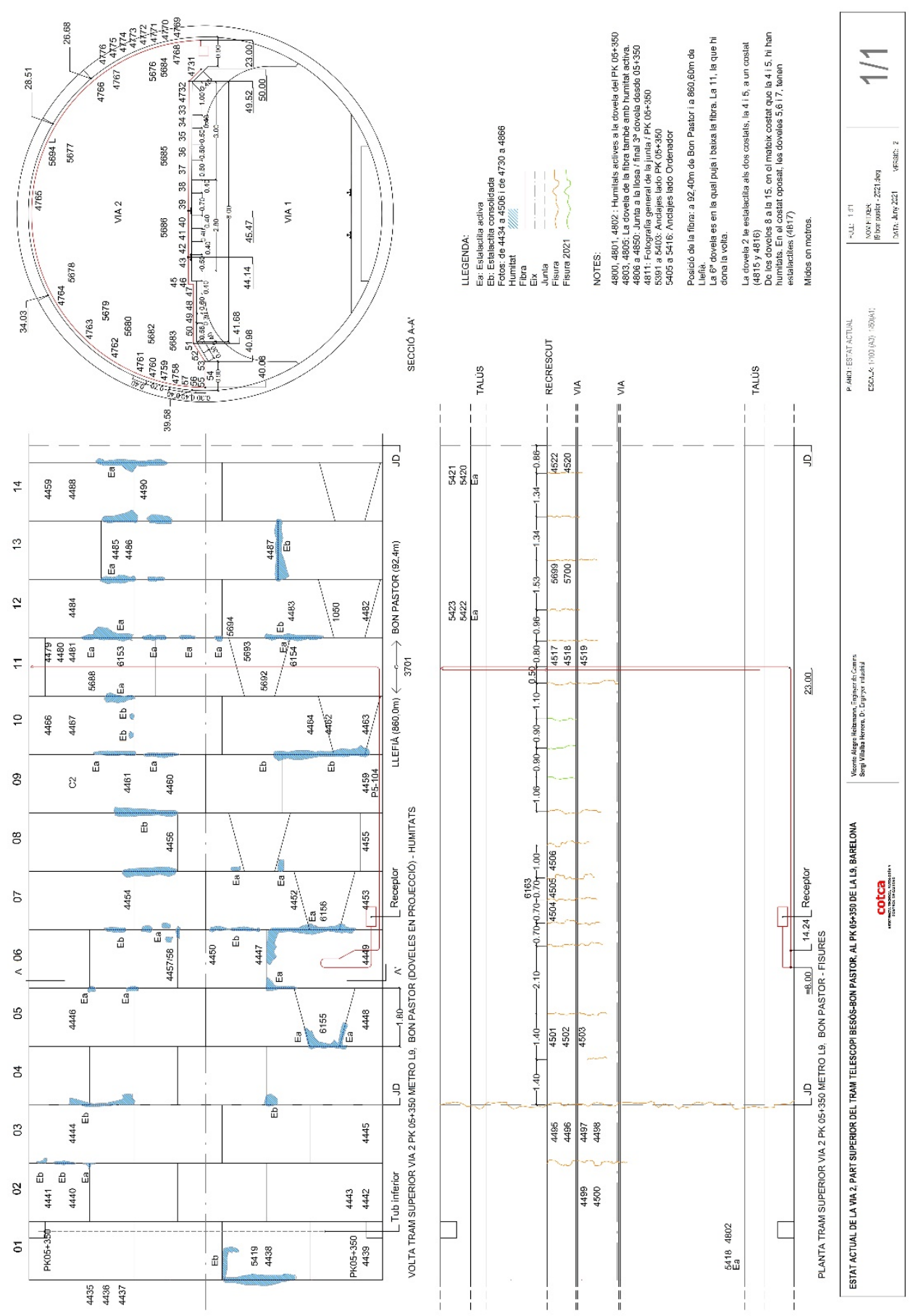

Figura 9. Plano estado actual.

En cada lectura posterior planteadas en las diferentes fases de la obra (previa al inicio de la obra, vaciado, finalización de la cimentación, estructura y fin de obra), se miden las microdeformaciones en los perímetros de las secciones transversales, se comparan con el modelo realizado y se emiten informes. Cuando se sobrepasen los criterios de ovalización prescritos en la normativa, o se detecten disfunciones, se actuará de acuerdo con el protocolo del plan de contingencias establecido.

Todo el sistema de monitorización se ha ubicado en la zona estratégica susceptible a la posible dinámica y evolución de la respuesta estructural (variaciones de microdeformaciones-tensiones, movimientos, posibles incrementos y/o desarrollo de mapas de fisuración, etc.) del túnel a lo 
largo de los trabajos de ejecución del nuevo edificio. Este sistema de instrumentación ha sido seleccionado considerando las condiciones de trabajo a las que estará sometido (variaciones de carga: descarga en la excavación y vaciado del terreno; y carga en la construcción del edificio). A continuación, se muestra el alcance de la monitorización utilizado (ver de la

Figura 10 a la Figura 13).

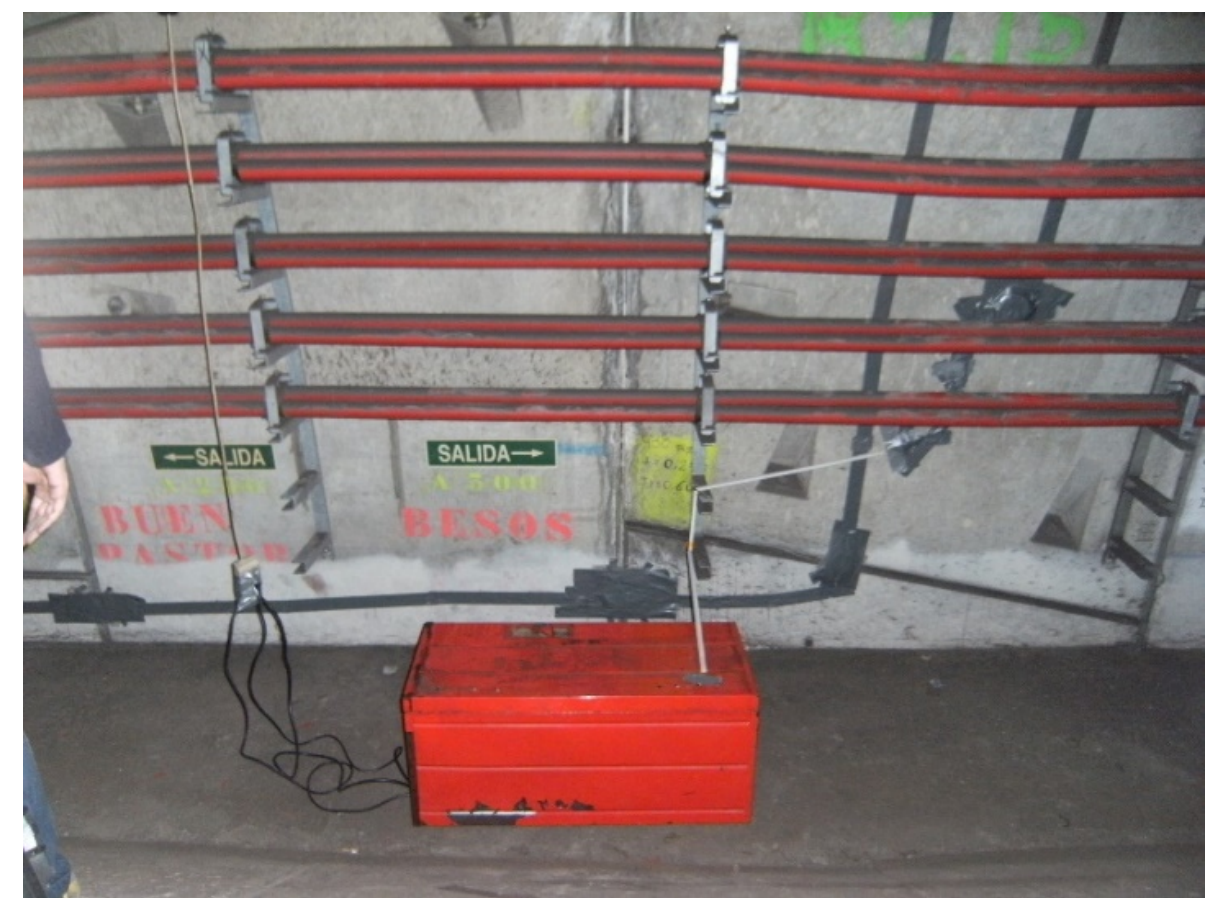

Figura 10. Instalación equipo de lectura de la fibra en lugar protegido.

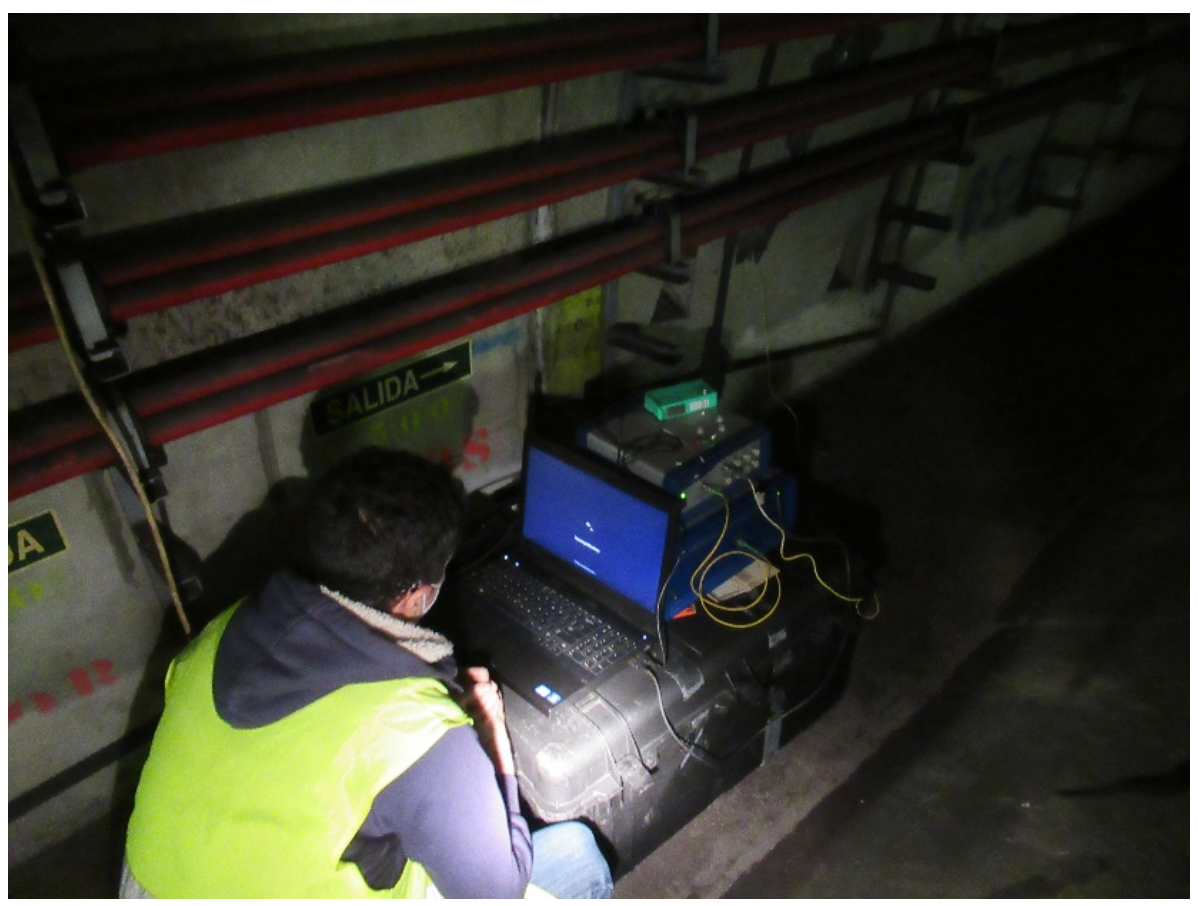

Figura 11. Recuperación de datos tomados por la fibra. 


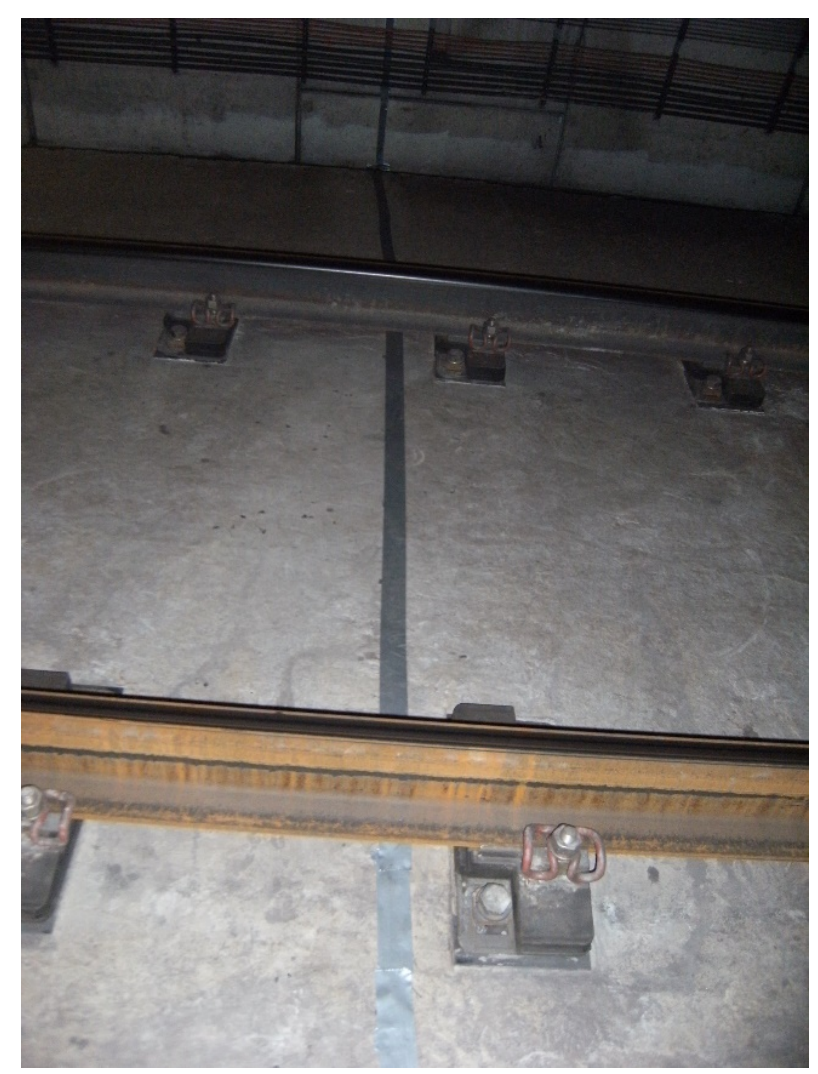

Figura 12. Fibra instalada y protegida en vía.

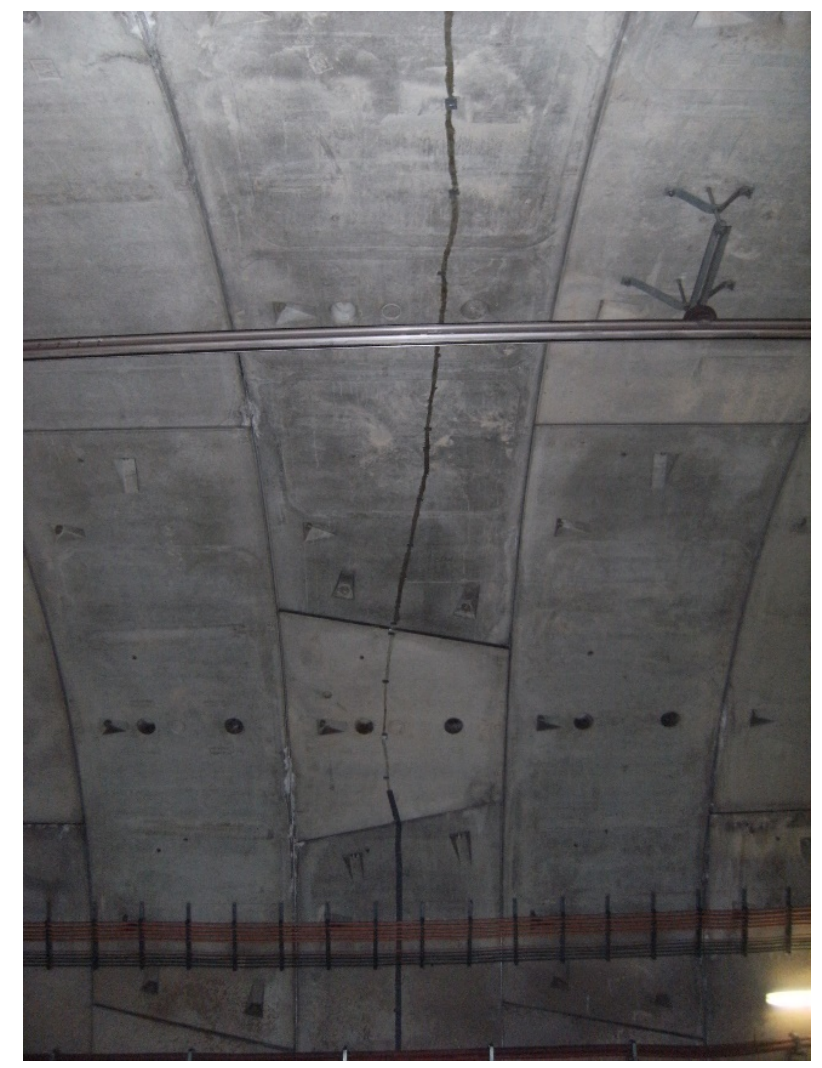

Figura 13. Fibra instalada y protegida en bóveda. 
El registro de las lecturas incorporadas en las gráficas corresponde a los días o las fases de obra en los que se realizaban operaciones de modificación de la situación o avance de la obra. Las fechas en que fue midiendo son las que se indican en la tabla (Tabla 2).

Tabla 2. Identificación de actuaciones y de sus períodos de tiempos.

\begin{tabular}{|c|c|}
\hline Período & Actuación \\
\hline 04 octubre 2018 & Estado Inicial. \\
\hline 07 octubre 2018 & Pre-excavación. \\
\hline $\begin{array}{cc}18 \text { octubre - } \\
\text { noviembre }\end{array}$ & Excavación. \\
\hline 13 diciembre 2018 & Ejecución parcial 1er tramo de la losa de cimentación (hasta junta J1). \\
\hline Período & Actuación \\
\hline 7 enero- 28 enero & $\begin{array}{l}\text { TJ } 1 \rightarrow \text { Hormigonado muros, pilares de planta PS-1 y encofrado TPS- } 1 \text {. } \\
\text { TJ } 2 \rightarrow \text { Hormigonado losa cimentación y armado + encofrado pilares de PS-1. } \\
\text { TJ } 3 \rightarrow \text { Sin ejecución. }\end{array}$ \\
\hline 28 enero-14 febrero & $\begin{array}{l}\text { TJ } 1 \rightarrow \text { Armado y hormigonado TPS-1. } \\
\text { TJ } 2 \rightarrow \text { Encofrado de forjado TPS-1. } \\
\text { TJ } 3 \rightarrow \text { Armado de losa cimentación. }\end{array}$ \\
\hline 14 febrero-18 marzo & $\begin{aligned} \text { TJ } 1 \rightarrow & \text { Armado, encofrado y hormigonado de pilares de PB. } \\
& \text { Encofrado de forjado TPB } \\
\text { TJ } 2 \rightarrow & \text { Armado y hormigonado de forjado TPS- } 1 \text {. } \\
& \text { Armado, encofrado y hormigonado de pilares de PB. } \\
\text { TJ } 3 \rightarrow \text { Hormigonado losa de cimentación. } & \text { Hormigonado muros, pilares de planta PS-1 }\end{aligned}$ \\
\hline 18 marzo-5 abril & $\begin{array}{l}\text { TJ } 1 \rightarrow \text { Hormigonado de forjado TPB y encofrado TP1. } \\
\text { TJ } 2 \rightarrow \text { Encofrado de forjado TPB. } \\
\text { TJ } 3 \rightarrow \text { Encofrado de forjado TPS- } 1 .\end{array}$ \\
\hline Período & Actuación \\
\hline 5 abril-18 abril & $\begin{array}{l}\text { TJ } 1 \rightarrow \text { Hormigonado de TP1. } \\
\text { TJ } 2 \rightarrow \text { Encofrado de forjado TPB (cont.). } \\
\text { TJ } 3 \rightarrow \text { Armado y hormigonado TPS- } 1 \text {. }\end{array}$ \\
\hline 18 abril-22 abril & Semana Santa \\
\hline 23 abril-10 mayo & $\begin{array}{l}\text { TJ } 1 \rightarrow \text { Hormigonado de TP1 (cont.). } \\
\text { TJ } 2 \rightarrow \text { Armado de forjado TPB } \\
\text { TJ } 3 \rightarrow \text { Armado y hormigonado TPS-1 (cont.). }\end{array}$ \\
\hline 10 mayo- 25 mayo & $\begin{array}{l}\text { TJ } 1 \rightarrow \text { Encofrado TP2. } \\
\text { TJ } 2 \rightarrow \text { Hormigonado de TPB. } \\
\text { TJ } 3 \rightarrow \text { Encofrado de forjado TPB. }\end{array}$ \\
\hline Período & Actuación \\
\hline 25 mayo-8 junio & $\begin{array}{l}\text { TJ } 1 \rightarrow \text { Hormigonado pilaresP2 + encofrado de TP2. } \\
\text { TJ } 2 \rightarrow \text { Hormigonado pilares P1. } \\
\text { TJ } 3 \rightarrow \text { Pilares PB y armado TPB. }\end{array}$ \\
\hline 8 junio-22 junio & $\begin{array}{l}\text { TJ } 1 \rightarrow \text { Hormigonado TP2. } \\
\text { TJ } 2 \rightarrow \text { Pilares P1. } \\
\text { TJ } 3 \rightarrow \text { Pantallas de PB a P1. }\end{array}$ \\
\hline 22 junio-6 julio & $\begin{array}{l}\text { TJ } 1 \rightarrow \text { Armado y hormigonado pilares P3. } \\
\text { TJ } 2 \rightarrow \text { Encofrado TP1 + Hormigonado escaleras de PB a P1. } \\
\text { TJ } 3 \rightarrow \text { Hormigonado TPB. }\end{array}$ \\
\hline 6 julio-20 julio & $\begin{array}{l}\text { TJ } 1 \rightarrow \text { Hormigonado Pantallas PB + hormigonado TP3. } \\
\text { TJ } 2 \rightarrow \text { Hormigonado de TP1 + pilares P2. } \\
\text { TJ } 3 \rightarrow \text { Pilares P1 + encofrado de forjado TP1. }\end{array}$ \\
\hline 20 julio-6 agosto & $\begin{array}{l}\text { TJ } 1 \rightarrow \text { Pilares P4. } \\
\text { TJ } 2 \rightarrow \text { Hormigonado TP2 + escaleras de P1 a P2. } \\
\text { TJ } 3 \rightarrow \text { Hormigonado TP1. }\end{array}$ \\
\hline
\end{tabular}


Para una mejor comprensión de las diferentes etapas y tareas realizadas, se incorporan los esquemas presentados en las figuras (Figura 14 y Figura 15) de fases con las juntas de dilatación de obra, que nos sirven como base de referencia de las tareas realizadas.

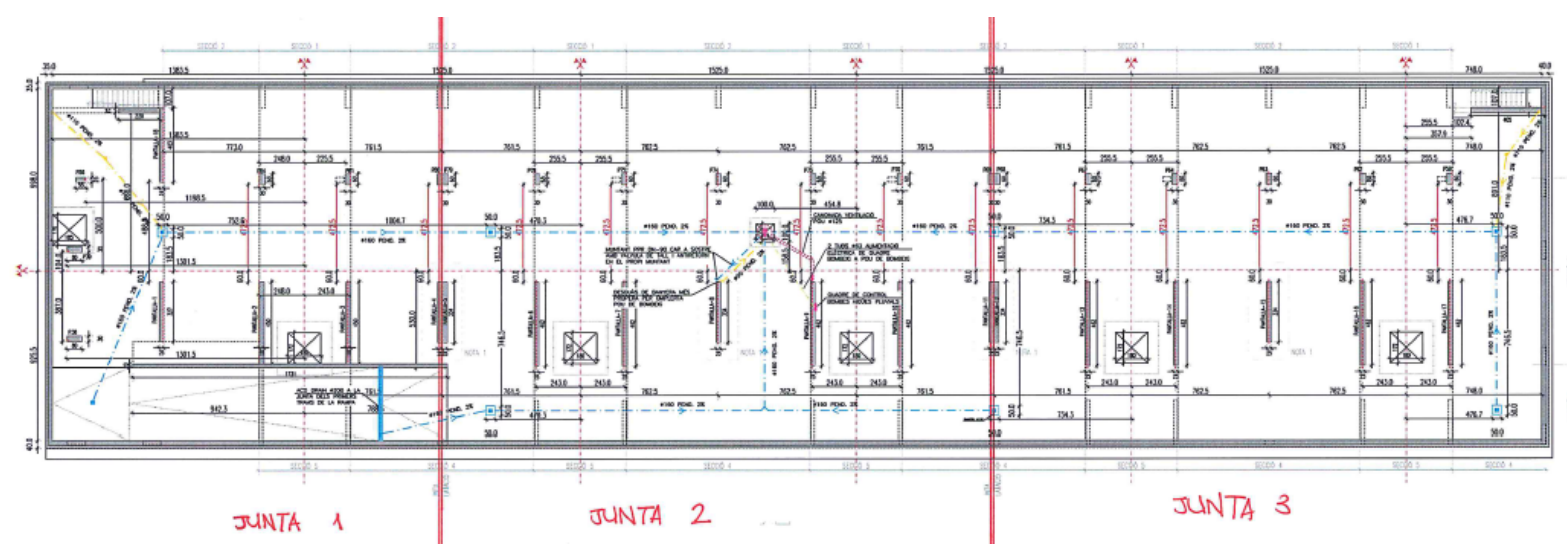

Figura 14. Planta del edificio en construcción.

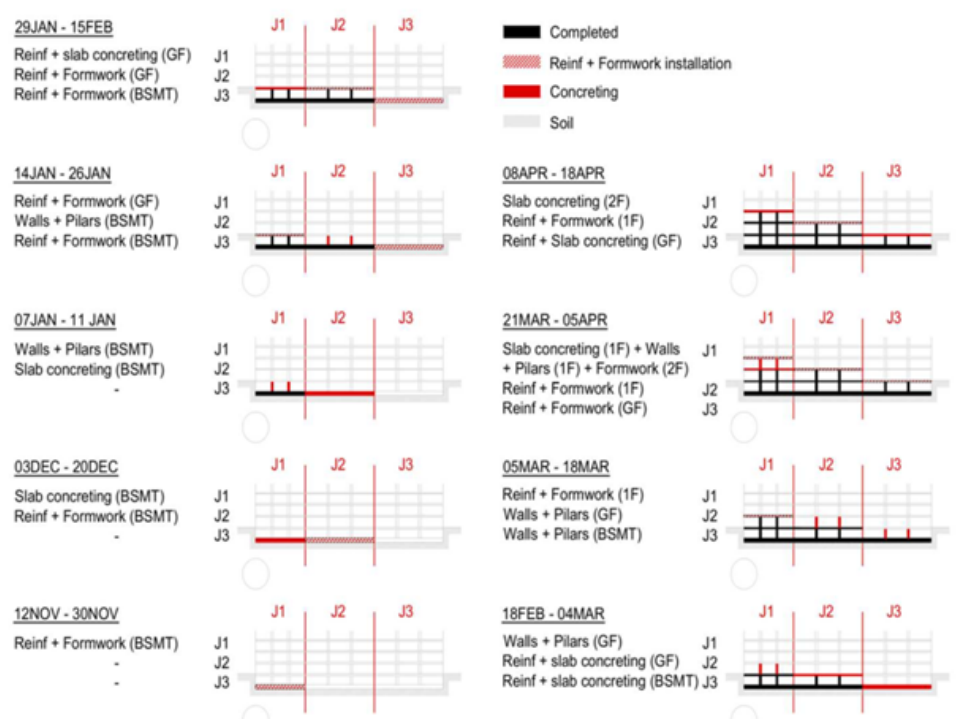

Los períodos de monitorización definidos con el objetivo de cubrir los períodos de construcción principales por separado: excavación, cimentación, ejecución de planta baja, primer piso, etc.

Intervalos de monitoreo:

Figura 15. Períodos de monitorización.

El modelo inicial (Figura 16) permitió considerar un plan de contingencias con avisos, alertas y alarmas que, una vez acabada la obra con la última medición, se puede ver si eran correctos o permitían un ajuste de la calibración gracias a los valores reales obtenidos en la ovalización del túnel en cada fase. También se puede ver que al final el aumento pequeño de microdeformaciones sobre la bóveda del túnel valida la actuación. 


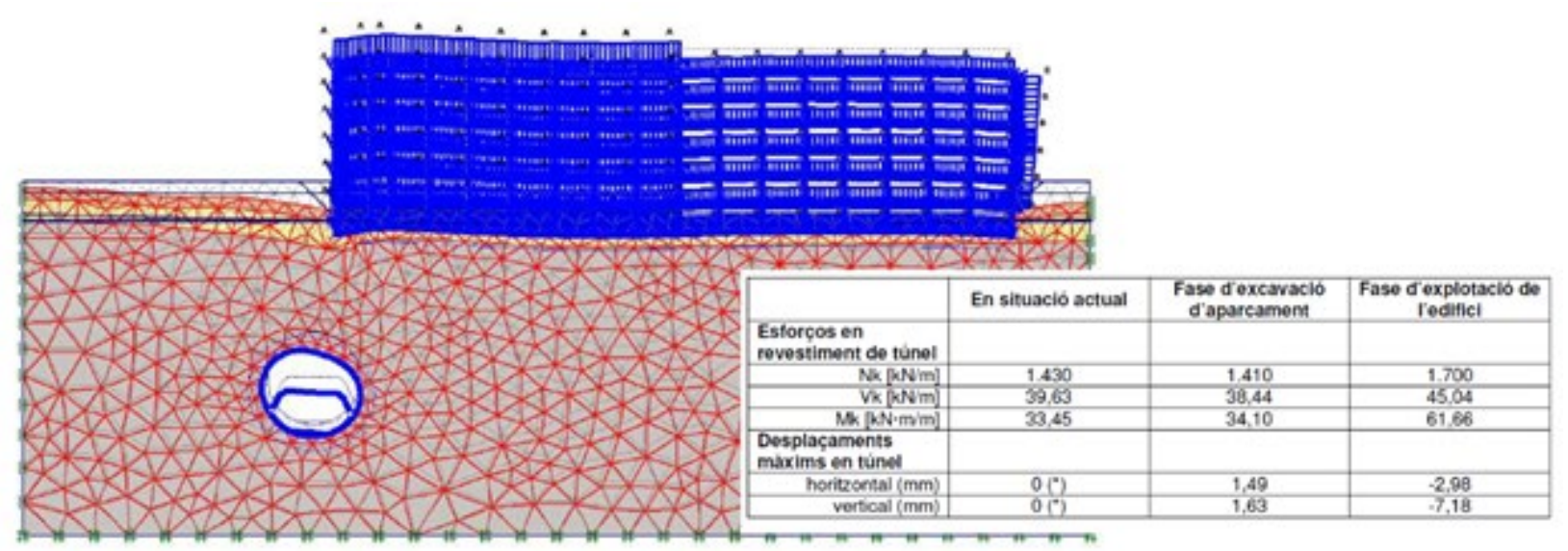

Figura 16. Modelo 3D (2D x metro lineal).

Si dentro de un año (2022) se vuelve a medir se tendrá la historia de cargas previa y se podrá evaluar si hay alguna nueva acción que produce algún tipo de deformación adicional. Con ello se está midiendo comportamientos reales que incorporan información del comportamiento estructural y permiten mejorar el modelo creando ese gemelo digital mejorado (DIGITAL TWIN). Mientras se mida de forma correcta y en los puntos idóneos de una infraestructura, se están reduciendo las incertidumbres y gestionando de una forma económica la obra realizada.

\section{CONCLUSIONES}

El Digital Twin (DT) es una tecnología prometedora que, recogiendo los datos de los ensayos de información y del comportamiento real de la estructura, crea una réplica virtual (de objetos y procesos) que simulan el comportamiento de sus homólogos reales en tiempo real y constituyen un "hermano gemelo" de lo realmente construido.

La fibra óptica es una herramienta muy útil para definir en el tiempo y en el espacio la respuesta de microdeformaciones del comportamiento de la estructura a lo largo de su vida útil, ya que en las secciones críticas se puede conocer el nivel de tensiones y el comportamiento térmico e incorporar esa información en el "gemelo digital" (DT).

La monitorización continua durante 8 meses (torre de refrigeración) y 29 meses (túnel) con fibra óptica ha ayudado a complementar los ensayos de información, a comprender la respuesta de la estructura y a avanzar en la creación de un gemelo digital (DT) basado en el comportamiento real de la estructura, que permite plantear posibles acciones de todo tipo y valorar el riesgo de cada una de ellas, en el "gemelo digital", antes de que ocurran en la realidad.

En el caso de la torre de refrigeración, la fibra permitió ayudar, con el resto de los ensayos de información, a calibrar el riesgo y plantear una reparación acorde con su respuesta para una vida útil determinada.

En el caso del túnel la fibra óptica permitió validar la ejecución de una obra sobre la bóveda y comprobar que no se afectaban los estados límites y de servicio al no superar los umbrales de riesgo preestablecidos. Por otra parte, una vez colocada en la estructura, la fibra óptica puede servir para futuras calibraciones del comportamiento del túnel. Los datos registrados permiten mejorar el modelo digital inicial, crear un "Digital Twin" que representa mejor el comportamiento del terreno y la rigidez real de la estructura, por ejemplo, modificando la rigidez del túnel con dovelas y calibrándola con la rigidez de la respuesta realmente obtenida de microdeformaciones en las fases de vaciado y puesta en carga.

Se han medido los parámetros que interesaban en unas secciones críticas en el tiempo y en el espacio para incorporarlas al gemelo digital, pero el mantenimiento de la obra, su gestión y la 
valoración del riesgo es una operación holística que debe sumar al seguimiento de estos parámetros otras medidas como la inspección periódica de la infraestructura y la instrumentación posterior de las posibles incidencias. Por ejemplo, en el caso del túnel, se pudo apreciar durante el seguimiento nocturno de las lecturas, que en la losa intermedia en la dirección transversal se producían nuevas fisuras bajo las vías que podrían estudiarse colocando la fibra óptica sobre la losa en dirección longitudinal para ver cuándo se producen (en qué momento preciso), estudiar la causa y valorar su riesgo, incorporando esa información en el futuro "gemelo digital” (Figura 17).
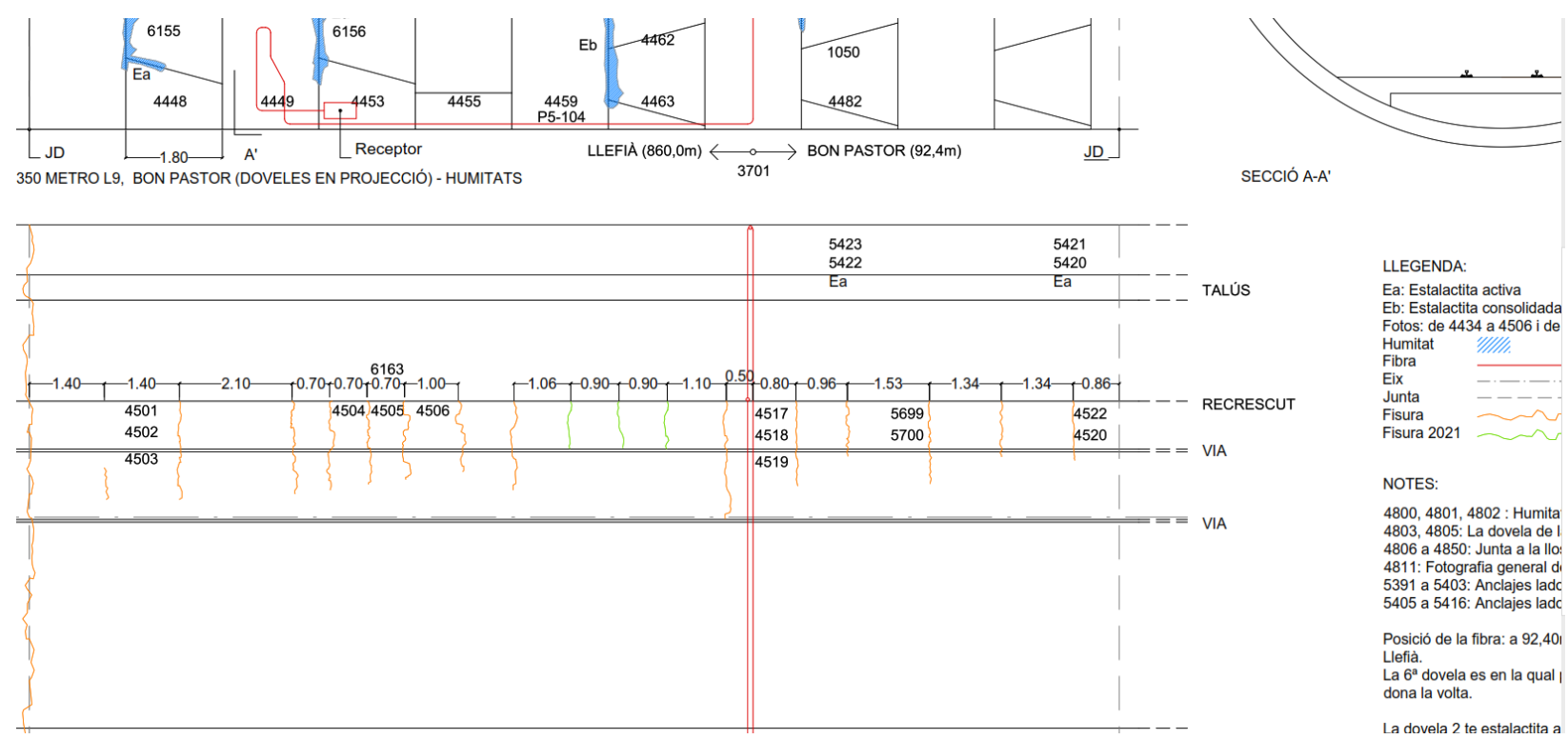

Figura 17. Detalle nuevas fisuras en la losa.

\section{AGRADECIMIENTOS}

Se agradece al Ingeniero de Transports Metropolitans de Barcelona (TMB) Ignacio Patón, y a Marta Marcet, arquitecta del Institut Municipal de l'Habitatge i Rehabilitació de Barcelona (IMHAB), las facilidades dadas para la toma de datos y obtención de información.

\section{REFERENCIAS}

(1) Rodríguez, G. (2017), "Monitorización de estructuras de hormigón mediante sensores de fibra óptica distribuida", Masters Thesis, Universitat Politècnica de Catalunya (UPC).

(2) Sachs, R. (May 2020). "Digital twins for construction" in: EUROSTRUCT, Live talk.

(3) Alegre, V., Villalba, S., (2019). "Avances en la instrumentación de estructuras con fibra óptica” in: CONPAT 2019.

(4) Alegre, V., Deulofeu, C., Patón, I., Ródenas, V. (2003). "Gestión de daños en infraestructuras" in: CONPAT 2003. 